\title{
Efficient Organic Light-Emitting Diodes with Fluorine-Doped Tin-Oxide Anode and Electrochemically Synthesized Sulfonated Polyaniline as Hole Transport Layer
}

\author{
Adriano R. V. Benvenho, José P. M. Serbena, Rudolf Lessmann, Ivo A. Hümmelgen, \\ Grupo de Dispositivos Optoeletrônicos Orgânicos, Departamento de Física, \\ Universidade Federal do Paraná, Caixa Postal 19044, 81531-990, Curitiba, Paraná, Brazil \\ Regina M. Q. de Mello, \\ Departamento de Química, Universidade Federal do Paraná, Caixa Postal 19044, 81531-990 Curitiba PR, Brazil \\ Rosamaria W. C. Li, Jamile H. Cuvero, and Jonas Gruber \\ Instituto de Química, Universidade de São Paulo, Caixa Postal 26077, 05513-970 São Paulo SP, Brazil
}

\author{
Received on 25 August, 2005
}

\begin{abstract}
In this work we report efficiency measurements on light-emitting diodes with electrochemically synthesized sulfonated polyaniline as hole transport layer. The anode used in our devices is fluorine-doped tin oxide, the blocking layer is electrochemically synthesized poly(9,9-dioctyl-1,4-fluorenylenevinylene) and the electron transporting material and emitter is tris-(8-hydroxyquinoline) aluminum. Sulfonated polyaniline based devices presented efficiency of $0.79 \mathrm{~cd} / \mathrm{A}$.
\end{abstract}

\section{INTRODUCTION}

Electroluminescent organic devices were first reported by Pope et al [1], who used anthracene as electroluminescent material. Helfrich et al [2] used the same material, but with different electrodes, achieving a smaller operation voltage. The major problem of these devices was the high voltage required for operation. The great development in this area began when Tang et al [3] and Burroughes et al [4] reported electroluminescence at low voltages in devices constructed with low molecular weight materials and polymeric materials, respectively. Since then, many different materials and device structures were tested and developed in order to improve device performance, increasing efficiency and lifetime.

Multilayer structures are the most used nowadays, because a correct choice of organic materials allows a better injection and recombination of charge carriers. Charge carriers that are unable to recombine emitting light, contribute to reduction of efficiency and loss of energy via heat dissipation [5]. Multilayered structures can be designed to present energy barriers inside the device, accumulating charges at the interfaces and increasing the recombination probability. In these structures the layer in contact with the high work function electrode must be a hole transporting material (HTM), and that one in contact with the low work function electrode must be an electron transporting material (ETM). The energy levels of these materials should almost match the work function of the electrodes to achieve a small injection barrier condition. The addition of a recombination or an exciton-blocking layer between these two layers increases the light emitted by the device, improving its efficiency [6].

Many conductive polymers have been used as HTM in light-emitting devices. Polyaniline [7,8] deposited by spin-coating belongs to the oldest. Polypyrrole was also used as HTM [9] in bilayer with MEH-PPV.
But, of the many soluble conductive polymers developed for this purpose so far, poly(3,4-ethylenedioxythiophene): poly(styrenesulfonate) (PEDOT:PSS), is one of the most investigated materials [10]. It is a polymeric blend, which can be processed easily because it is dispersed in water. It was observed that PEDOT:PSS reduces the effective energy barrier for charge injection into the organic electroluminescent material $[11,12]$ leading to an improvement of efficiency when using this material as HTM in OLEDs.

Sulfonated polyaniline (SPAN) is a self-doped conductive polymer, dispensing the need of blending with a doping material. SPAN synthesis and electrical behavior were investigated by different groups $[13,14,15]$ and Onoda et al [16] reported the application of SPAN to OLEDs. SPAN makes ohmic contact with different metals [17] and for this reason it was proposed as hole injection material [18]. SPAN can be chemically or electrochemically synthesized. The advantage of the electrochemical synthesis when compared to the spin-coating deposition is the lower waste of material, the formation of a well-adsorbed and mechanically tough film, direct deposition on desired substrate and possibility to control different parameters during polymerization. As a single component HTM, it is also not subjected to phase segregation. In this work we determine the efficiency of devices constructed with electrochemically synthesized SPAN and compare it with devices made with PEDOT:PSS, as a reference.

\section{EXPERIMENTAL}

The electroluminescent devices were constructed in sandwich structure. Glass covered with fluorine-doped tin oxide (FTO) supplied by Vidcon Ltda $(12 \Omega / \square)$ was used as substrate and anode.

The electrochemical synthesis of SPAN was performed in a 
conventional three-electrode electrochemical cell, containing FTO working electrode, graphite as counter electrode, and a saturated $\mathrm{Ag} / \mathrm{AgCl}$ reference electrode. SPAN film formation was carried out by cycling the potential between -0.15 and $1.10 \mathrm{~V}$ at $100 \mathrm{mV} / \mathrm{s}$ in $0.1 \mathrm{~mol} / \mathrm{L}$ camphorsulfonic acid (CSA) $+0.1 \mathrm{~mol} / \mathrm{L}$ metanilic acid ( $m$-aminobenzenesulfonic acid) + $0.01 \mathrm{~mol} / \mathrm{L}$ aniline aqueous electrolytic solution [17]. Electrochemical polymerization was performed for 10, 20, 30, 40 or 50 cycles.

PEDOT:PSS (Baytron P) was deposited onto FTO from water solution as received and, in the sequence, annealed at 60 ${ }^{o} \mathrm{C}$ during 3 hours in vacuum.

Poly(9,9-dioctyl-1,4-fluorenylenevinylene), PDO14FV [19] was used as electron blocking layer and tris-(8hydroxyquinoline) aluminum, $\mathrm{Alq}_{3}$, as ETM. PDO14FV was synthesized electrochemically, as fully described elsewhere [19]. It was dissolved in $\mathrm{CHCl}_{3}(6 \mathrm{mg} / \mathrm{mL})$ and deposited by spin-coating ( $\sim 23 \mathrm{~nm}$ ) onto SPAN or PEDOT:PSS film. $\mathrm{Alq}_{3}$ was purchased from Sigma Aldrich and used as received. The $\mathrm{Alq}_{3}$ layer $(\sim 48 \mathrm{~nm})$ was deposited by sublimation at a pressure of $\sim 10^{-6}$ torr. The chemical structures of these materials are shown in Fig. 1. Ca and $\mathrm{Al}$ were evaporated at a pressure of $\sim 10^{-6}$ torr. The thickness of the layers was measured using a Dektak3surface profiler. The devices were encapsulated using the procedure described by Toniolo et al [20]. The current density versus voltage $(J \times V)$ curves were measured using a Keithley 230 voltage source and a Keithley 2010 multimeter. The luminance versus voltage $(L \times V)$ measurements were made with a calibrated photodiode, OSD15-5T (Centronic), and a Keithley 6485 picoammeter. The electroluminescence curves were recorded using a Hitachi F4500 fluorescent spectrophotometer.

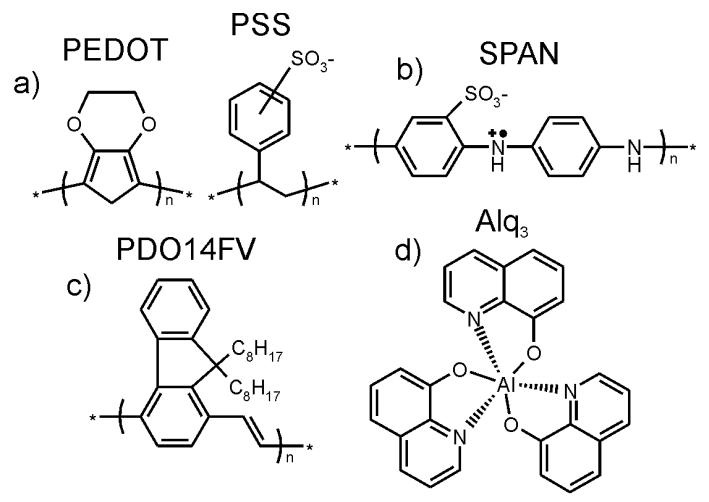

FIG. 1: Chemical structures of the used materials: a) PEDOT:PSS; b) SPAN; c) PDO14FV; d) Alq .

\section{RESULTS AND DISCUSSION}

The energy level diagram of the used materials, considering isolated materials, based on values obtained from the literature [16,19,21,22], is presented in Fig. 2.

The $J \times V$ results presented in Fig. 3 correspond to devices assembled with different SPAN layers. Results of de-

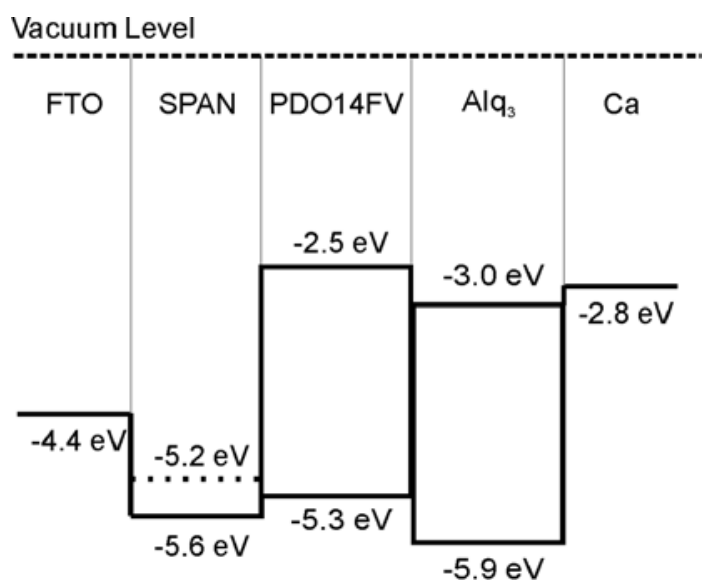

FIG. 2: Band diagram of materials based on literature values. The dotted line represents the HOMO level of PEDOT:PSS.

vices based on PEDOT:PSS (circles) as HTM were included for comparison. Devices with this PEDOT:PSS layer thickness showed the highest efficiency among those we have prepared with PEDOT:PSS.

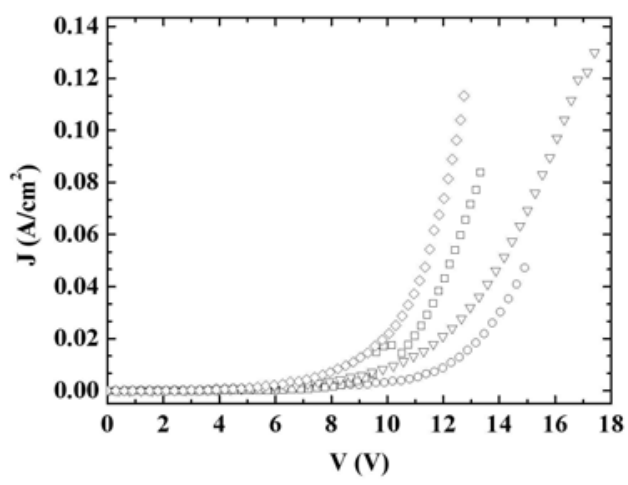

FIG. 3: Current density $\times$ voltage characteristic curves of (circles): FTO / SPAN(40 nm) / PDO14FV / $\mathrm{Alq}_{3} / \mathrm{Ca} / \mathrm{Al}$; (down triangle): FTO / SPAN(74 nm) / PDO14FV / Alq 3 / Ca/Al; (diamond): FTO / SPAN(131 nm)/PDO14FV/Alq 3 / Ca / Al; (squares): FTO / PE$\mathrm{DOT}(100 \mathrm{~nm}) / \mathrm{PDO} 14 \mathrm{FV} / \mathrm{Alq}_{3} / \mathrm{Ca} / \mathrm{Al}$.

Figure 4 presents the $L \times V$ curves of the devices whose $J \times V$ results are presented in Fig. 3. Luminescence is not observed at reverse bias and for this reason reverse bias data are omitted in all figures. At forward bias all devices emit light, reaching a maximum of $500 \mathrm{~cd} / \mathrm{m}^{2}$ for SPAN $(40 \mathrm{~nm}$ thick).

It was demonstrated that the introduction of PEDOT:PSS as intermediate layer between the anode and the electroluminescent polymer effectively eliminates the potential barrier existing between the anode and PEDOT:PSS, so that the remaining effective barrier is only that existing between PEDOT:PSS and 


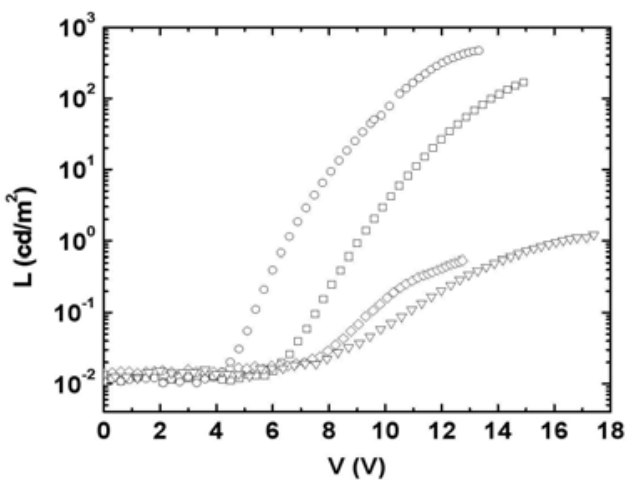

FIG. 4: Luminance $\times$ voltage curves of (circles): FTO / SPAN(40 $\mathrm{nm}$ ) / PDO14FV / Alq ${ }_{3} / \mathrm{Ca} / \mathrm{Al}$; (down triangle): FTO / SPAN(74 $\mathrm{nm}$ ) / PDO14FV / Alq 3 / Ca / Al; (diamond): FTO / SPAN(131 nm) / PDO14FV / $\mathrm{Alq}_{3} / \mathrm{Ca} / \mathrm{Al}$; (squares): FTO / PEDOT(100 nm) / $\mathrm{PDO} 14 \mathrm{FV} / \mathrm{Alq}_{3} / \mathrm{Ca} / \mathrm{Al}$.

the electroluminescent polymer [23]. SPAN was proposed to be used with the same intent [18]. The fact that better performances are achieved with thinner SPAN layers than PEDOT:PSS layers suggests that SPAN presents a larger polarization or charge displacement capability than PEDOT:PSS, which leads to the energy levels equalization and elimination of interfacial energy barrier even with thinner layers. This could explain the almost similar current densities even knowing that SPAN has a larger (more negative) highest occupied molecular orbital energy level, as represented in Fig. 2. However, a thicker SPAN layer could constrain the charge carrier transport from the electrode, diminishing the injected carriers into PDO14FV, due to its higher resistance.

The calculated current efficiencies versus current density are shown in Fig. 5. As can be seen, the $40 \mathrm{~nm}$ thick SPANbased device presents higher efficiency over all the current density interval. The maximum value for SPAN-based devices is $0.79 \mathrm{~cd} / \mathrm{A}$ at $14.5 \mathrm{~mA} / \mathrm{cm}^{2}$. So, although the devices presented similar brightness, the SPAN-based one is still more efficient.

The electroluminescence spectra of FTO / SPAN / PDO14FV / $\mathrm{Alq}_{3}$ / Ca / Al; FTO / PEDOT:PSS / PDO14FV / $\mathrm{Alq}_{3} / \mathrm{Ca} / \mathrm{Al}$; $\mathrm{FTO} / \mathrm{PEDOT} / \mathrm{Alq}_{3} / \mathrm{Ca} / \mathrm{Al}$ and FTO / PEDOT:PSS / PDO14FV / Ca / Al devices are presented in Fig. 6. It can be seen that devices containing PDO14FV and $\mathrm{Alq}_{3}$ layers show an electroluminescence spectrum analogous of device without PDO14FV layer, indicating that the dominant emission is that occurring in the $\mathrm{Alq}_{3}$ layer. This point is important since, despite the fact that the peak of emission of PDO14FV is almost at the same wavelength of $\mathrm{Alq}_{3}$, PDO14FV presents a broader emission spectrum, which leads to significantly different CIE coordinates, as can be seen in Fig. 7.

The results presented above demonstrate that SPAN effectively favors the injection of positive charge carriers into

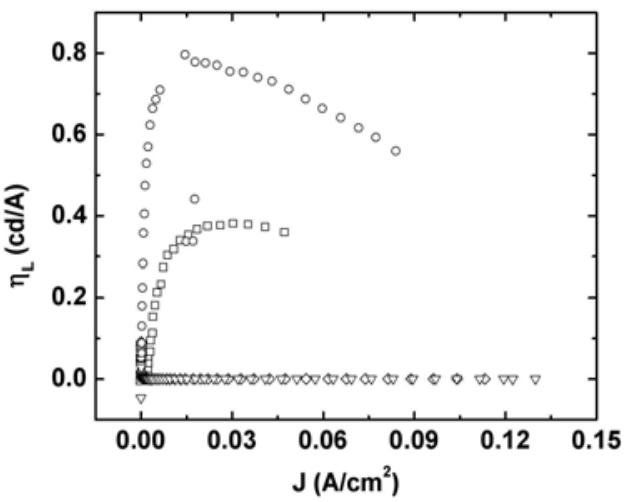

FIG. 5: Current efficiency $\times$ current density of (circles): FTO / $\mathrm{SPAN}(40 \mathrm{~nm}) / \mathrm{PDO} 14 \mathrm{FV} / \mathrm{Alq}_{3} / \mathrm{Ca} / \mathrm{Al}$; (down triangle): $\mathrm{FTO}$ / SPAN(74 nm) / PDO14FV / Alq 3 / Ca / Al; (diamond): FTO / $\mathrm{SPAN}(131 \mathrm{~nm}) / \mathrm{PDO} 14 \mathrm{FV} / \mathrm{Alq}_{3} / \mathrm{Ca} / \mathrm{Al}$; (squares): FTO / PEDOT(100 nm) / PDO14FV / Alq 3 / Ca / Al.

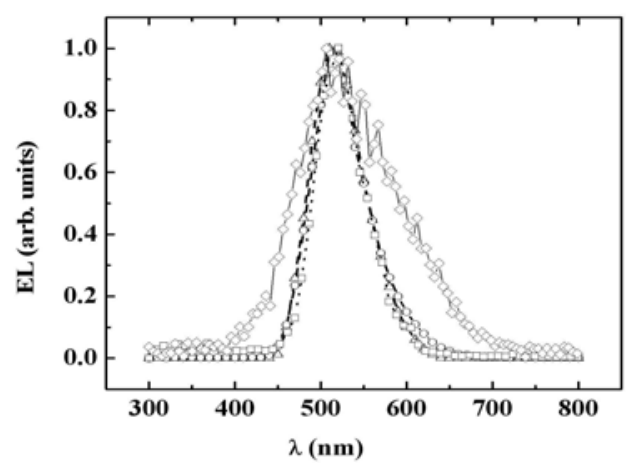

FIG. 6: Electroluminescence spectrum of : (up triangles): FTO / SPAN / PDO14FV / $\mathrm{Alq}_{3}$ / Ca / Al device; (circles): FTO / PEDOT:PSS / PDO14FV / Alq / Ca / Al device; (squares): FTO / PEDOT / $\mathrm{Alq}_{3}$ / Ca / Al device; (diamonds): FTO / PEDOT:PSS / $\mathrm{PDO} 14 \mathrm{FV} / \mathrm{Ca} / \mathrm{Al}$ device.

PDO14FV. The transport of these carriers through the device is then hindered at the interface with $\mathrm{Alq}_{3}$ (see Fig. 2), due to the presence of the energy barrier, avoiding that positive charge carriers simply pass through the device without pair formation and recombination possibility. At the other electrode interface, Ca provides easy electron injection, due to the absence of an energy barrier hindering this process. The injected electrons are transported through the $\mathrm{Alq}_{3}$, accumulating at the $\mathrm{Alq}_{3} / \mathrm{PDO} 14 \mathrm{FV}$ interface, due to the energy barrier for electrons at this interface. At this PDO14FV/Alq 3 interface, there is then a high holes/electrons concentration, respectively. Since the emission occurs predominantly in the $\mathrm{Alq}_{3}$, it means that either the holes transport through these 


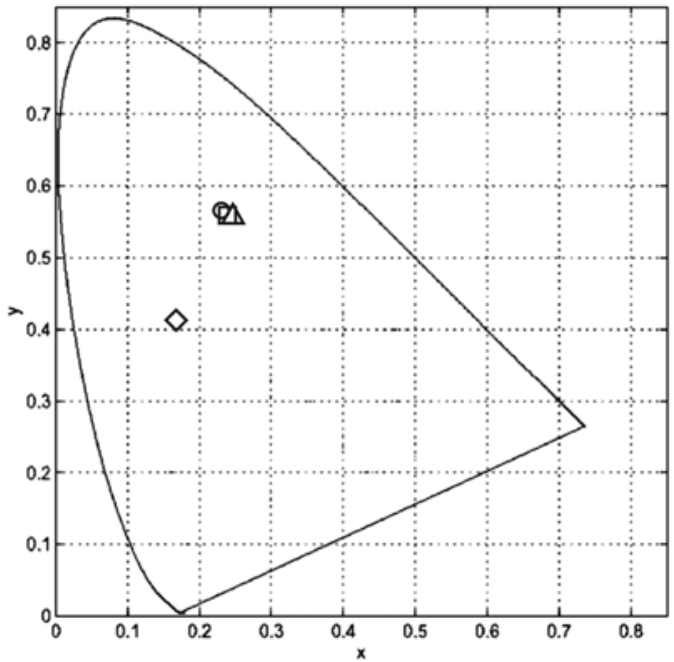

FIG. 7: CIE coordinates of (up triangle): FTO / SPAN / PDO14FV / $\mathrm{Alq}_{3} / \mathrm{Ca} / \mathrm{Al}$ device $(x=0.23 ; \mathrm{y}=0.58)$; (circle): $\mathrm{FTO} / \mathrm{PE}-$ DOT:PSS / PDO14FV / $\mathrm{Alq}_{3} / \mathrm{Ca} / \mathrm{Al}$ device $(x=0.25 ; \mathrm{y}=0.57)$; (square): FTO / PEDOT / $\mathrm{Alq}_{3} / \mathrm{Ca} / \mathrm{Al}$ device $(\times=0.23 ; \mathrm{y}=0.58)$; (diamonds): FTO / PEDOT:PSS / PDO14FV / Ca / Al device $(\times=$ $0.17 ; \mathrm{y}=0.43)$. interface is easier than electron transport in the opposite direction and so, pair generation and recombination occurs in the $\mathrm{Alq}_{3}$ or; that pairs generated in the PDO14FV tend to be transferred to the $\mathrm{Alq}_{3}$. The second process is improbable assuming the correctness of the band structure presented in Fig. 2 , because it would imply in pair energy increase, if additional relaxation phenomena would not be present.

\section{CONCLUSION}

In this work we reported the construction of light-emitting diodes (OLEDs) using electrochemically synthesized sulfonated polyaniline (SPAN) as HTM. The best performance was obtained with $40 \mathrm{~nm}$ thick SPAN layer. This SPANbased device presented a higher current efficiency, reaching $0.79 \mathrm{~cd} / \mathrm{A}$ at $14.5 \mathrm{~mA} / \mathrm{cm}^{2}$.

Our work demonstrates that efficient OLEDs can be constructed using electrochemically synthesized polymers. SPAN is directly synthesized onto the anode material and PDO14FV is prepared by controlled potential electrolysis $[19,24]$ and further deposited by spin-coating technique.

\section{Acknowledgements}

The authors would like to thank CAPES, CNPq, FAPESP and CT-ENERG/CNPq for research grants.
[1] M. Pope, H. Kallmann, and P. Magnante, J. Chem. Phys. 38, 2042 (1963).

[2] W. Helfrich, W. G. Schneider, Phys. Rev. Lett. 14, 229 (1965).

[3] C. W. Tang, S. A. Van Slyke, Appl. Phys. Lett. 51, 913 (1987).

[4] J. H. Burroughes, D. D. C. Bradley, A. R. Brown. R. N. Marks, K. Mackay, R. H. Friend, P. L. Burns, and A. B. Holmes, Nature 347, 539 (1990).

[5] C. Adachi, T. Tsutsui, in Organic Light Emitting Devices, edited by J. Shinar, Springer, 2003.

[6] W. R. Salaneck, K. Seki, A. Kahn, and J. J. Pireaux, Conjugated Polymers and Molecular Interfaces, 2000, Marcel Dekker, Inc.

[7] G. Gustafsson, Y. Cao, G. M. Treacy, F. Klavetter, N. Colaneri, and A. J. Heeger, Nature 357, 477 (1992).

[8] Y. Yang, A. J. Heeger, Appl. Phys. Lett. 64, 1245 (1994).

[9] J. Gao, A. J. Heeger, J. Y. Lee, and C. K. Kim, Synth. Met. 82, 221 (1996)

[10] Y. Cao , G. Yu , C. Zhang, R. Menon, and A. J. Heeger, Synth. Met. 87, 171 (1997).

[11] N. Koch, A. Kahn, J. Ghijsen, J. J. Pireaux, J. Schwartz, R. L. Johnson, and A. Elschner, Appl. Phys. Lett. 82, 70 (2003).

[12] G. Greczynski , T. Kugler, M. Keil , W. Osikowicz , M. Fahlman, and W.R. Salaneck, J. Electron. Spectrosc. Relat. Phenom. 121, 1 (2001).
[13] W. Lee, G. Du, S. M. Long, A. J. Epstein, S. Shimizu, T. Saitoh, and M. Uzawa, Synth. Met. 84, 807 (1997).

[14] I. Mav, M. Zigon, and A. Sebenik, Synth. Met. 101, 717 (1999).

[15] W. P. Lee, K. R. Brenneman, C. H. Hsu, H. Shih, and A. J. Epstein, Macromolecules 34, 2648 (2001).

[16] M. Onoda, K. Yoshino, Jpn. J. Appl. Phys. 34, L260 (1995).

[17] R. M. Q. Mello, I. A. Hümmelgen, J. Solid State Electrochem. 5, 546 (2001).

[18] L. S. Roman, R. M. Q. Mello, F. Cunha, and I. A. Hümmelgen, J. Solid State Electrochem. 8, 118 (2004).

[19] J. Gruber, R. W. C. Li, L. H. J. M. C. Aguiar, A. R. V. Benvenho, R. Lessmann, and I. A. Hümmelgen, J. Mater. Chem. 15, 517 (2005).

[20] R. Toniolo, I. A. Hümmelgen, Macromol. Mater. Eng. 289, 311 (2004).

[21] J. H. Yang, K. C. Gordon, Chem. Phys. Lett. 375, 649 (2003).

[22] A. Andersson, N. Johansson, P. Bröms, N. Yu, D. Lupo, and W. R. Salaneck, Adv. Mat. 10, 859 (1998).

[23] T. Kugler, W. R. Salaneck, H. Rost, and A. B. Holmes, Chem. Phys. Lett. 310, 391 (1999).

[24] J. H. P. Utley, Y. P. Gao, J. Gruber, Y. Zhang, and A. MunozEscalona, J. Mater. Chem. 5, 1297 (1995). 